\title{
A Semantic Scattering Model for the Automatic Interpretation of Genitives
}

\author{
Dan Moldovan \\ Language Computer Corporation \\ Richardson, TX 75080 \\ Adriana Badulescu \\ Language Computer Corporation \\ Richardson, TX 75080 \\ moldovan@languagecomputer.com adriana@languagecomputer.com
}

\begin{abstract}
This paper addresses the automatic classification of the semantic relations expressed by the English genitives. A learning model is introduced based on the statistical analysis of the distribution of genitives' semantic relations on a large corpus. The semantic and contextual features of the genitive's noun phrase constituents play a key role in the identification of the semantic relation. The algorithm was tested on a corpus of approximately 2,000 sentences and achieved an accuracy of $79 \%$, far better than $44 \%$ accuracy obtained with C5.0, or $43 \%$ obtained with a Naive Bayes algorithm, or $27 \%$ accuracy with a Support Vector Machines learner on the same corpus.
\end{abstract}

\section{Introduction}

\subsection{Problem Description}

The identification of semantic relations in open text is at the core of Natural Language Processing and many of its applications. Detecting semantic relations is useful for syntactic and semantic analysis of text and thus plays an important role in automatic text understanding and generation. Furthermore, semantic relations represent the core elements in the organization of lexical semantic knowledge bases used for inferences. Recently, there has been a renewed interest in text semantics fueled in part by the complexity of some major research initiatives in Question Answering, Text Summarization, Text Understanding and others, launched in the United States and abroad.

Two of the most frequently used linguistic constructions that encode a large set of semantic relations are the s-genitives, e.g. "man's brother", and the of-genitives, e.g. "dress of silk". The interpretation of these phrase-level constructions is paramount for various applications that make use of lexical semantics.

Example: "The child's mother had moved the child from a car safety seat to an area near the open passenger-side door of the car." (The Desert Sun, Monday, October 18th, 2004).

There are two semantic relations expressed by genitives: (1) "child's mother" is an s-genitive encoding a KINSHIP relation, and (2) "passenger-side door of the car" is an of-genitive encoding a PARTWHOLE relation.

This paper provides a detailed corpus analysis of genitive constructions and a model for their automatic interpretation in English texts.

\subsection{Semantics of Genitives}

In English there are two kinds of genitives. In general, in one, the modifier is morphologically linked to the possessive clitic 's and precedes the head noun (s-genitive, i.e. $N P_{\text {modif }}$ 's $\left.N P_{\text {head }}\right)$, and in the second one the modifier is syntactically marked by the preposition of and follows the head noun (ofgenitive, i.e. $N P_{\text {head }}$ of $\left.N P_{\text {modif }}\right)$.

Although the genitive constructions have been studied for a long time in cognitive linguistics, their semantic investigation proved to be very difficult, as 
the meanings of the two constructions are difficult to pin down. There are many factors that contribute to the genitives' semantic behavior, such as the type of the genitive, the semantics of the constituent nouns, the surrounding context, and others.

A characteristic of genitives is that they are very productive, as the construction can be given various semantic interpretations. However, in some situations, the number of interpretations can be reduced by employing world knowledge. Consider the examples, "Mary's book" and "Shakespeare's book". "Mary's book" can mean the book Mary owns, the book Mary wrote, the book Mary is reading, or the book Mary is very fond of. Each of these interpretations is possible in the right context. In "Shakespeare's book", however, the preferred interpretation, provided by a world knowledge dictionary, is the book written by Shakespeare.

\subsection{Previous Work}

There has been much interest recently on the discovery of semantic relations from open-text using symbolic and statistical techniques. This includes the seminal paper of (Gildea and Jurafsky, 2002), Senseval 3 and coNLL competitions on automatic labeling of semantic roles detection of noun compound semantics (Lapata, 2000), (Rosario and Hearst, 2001) and many others. However, not much work has been done to automatically interpret the genitive constructions.

In 1999, Berland and Charniak (Berland and Charniak, 1999) applied statistical methods on a very large corpus to find PART-WHOLE relations. Following Hearst's method for the automatic acquisition of hypernymy relations (Hearst, 1998), they used the genitive construction to detect PARTWHOLE relations based on a list of six seeds representing whole objects, (i.e. book, building, car, hospital, plant, and school). Their system's output was an ordered list of possible parts according to some statistical metrics (Dunning's log-likelihood metric and Johnson's significant-difference metric). They presented the results for two specific patterns ( " $N N$ 's $N N$ " and "NN of DT NN"). The accuracy obtained for the first 50 parts was $55 \%$ and for the first 20 parts was $70 \%$.

In 2003, Girju, Badulescu, and Moldovan (Girju, Badulescu, and Moldovan, 2003) detected the PART-
WHOLE relations for some of the most frequent patterns (including the genitives) using the Iterative Semantic Specialization, a learning model that searches for constraints in the WordNet noun hierarchies. They obtained an f-measure of $93.62 \%$ for sgenitives and $91.12 \%$ for of-genitives for the PARTWHOLE relation.

Given the importance of the semantic relations encoded by the genitive, the disambiguation of these relations has long been studied in cognitive linguistics (Nikiforidou, 1991), (Barker, 1995), (Taylor, 1996), (Vikner and Jensen, 1999), (Stefanowitsch, 2001), and others.

\section{Genitives' Corpus Analysis}

\subsection{The Data}

In order to provide a general model of the genitives, we analyzed the syntactic and semantic behavior of both constructions on a large corpus of examples selected randomly from an open domain text collection, LA Times articles from TREC-9. This analysis is justified by our desire to answer the following questions: "What are the semantic relations encoded by the genitives?" and "What is their distribution on a large corpus?"

A set of 20,000 sentences were randomly selected from the LA Times collection. In these 20,000 sentences, there were 3,255 genitive instances $(2,249$ of-constructions and 1,006 s-constructions). From these, $80 \%$ were used for training and $20 \%$ for testing.

Each genitive instance was tagged with the corresponding semantic relations by two annotators, based on a list of 35 most frequently used semantic relations proposed by (Moldovan et al., 2004) and shown in Table 1. The genitives' noun components were manually disambiguated with the corresponding WordNet 2.0 senses or the named entities if they are not in WordNet (e.g. names of persons, names of locations, etc).

\subsection{Inter-annotator Agreement}

The annotators, two graduate students in Computational Semantics, were given the genitives and the sentences in which they occurred. Whenever the annotators found an example encoding a semantic relation other than those provided, they had to tag it as "OTHER". Besides the type of relation, the an- 
notators were asked to provide the correct WordNet 2.0 senses of the two nouns and information about the order of the modifier and the head nouns in the genitive construction. For example, although in ofconstructions the head is followed by the modifier most of the time, this is not always true. For instance, in "owner of car[POSSESSION]" the head owner is followed by the modifier car, while in "John's car[POSSESSION/R]" the order is reversed. Approximately one third of the training examples had the nouns in reverse order.

Most of the time, one genitive instance was tagged with one semantic relation, but there were also situations in which an example could belong to more than one relation in the same context. For example, the genitive "city of USA" was tagged as a PARTWHOLE relation and as a LOCATION relation. There were 21 such cases in the training corpus.

The judges' agreement was measured using the Kappa statistics (Siegel and Castelan, 1988), one of the most frequently used measure of interannotator agreement for classification tasks: $K=$ $\frac{\operatorname{Pr}(A)-\operatorname{Pr}(E)}{1-\operatorname{Pr}(E)}$, where $\operatorname{Pr}(A)$ is the proportion of times the raters agree and $\operatorname{Pr}(E)$ is the probability of agreement by chance.

The $\mathrm{K}$ coefficient is 1 if there is a total agreement among the annotators, and 0 if there is no agreement other than that expected to occur by chance.

On average, the $\mathrm{K}$ coefficient is close to 0.82 for both of and s-genitives, showing a good level of agreement for the training and testing data on the set of 35 relations, taking into consideration the task difficulty. This can be explained by the instructions the annotators received prior to annotation and by their expertise in lexical semantics.

\subsection{Distribution of Semantic Relations}

Table 1 shows the distribution of the semantic relations in the annotated corpus.

In the case of of-genitives, there were 19 relations found from the total of 35 relations considered. The most frequently occurring relations were POSSESSION, KINSHIP, PROPERTY, PART-WHOLE, LOCATION, SOURCE, THEME, and MEASURE.

There were other relations (107 for of-genitives) that do not belong to the predefined list of 35 relations, such as "state of emergency". These examples were clustered in different undefined subsets based

\begin{tabular}{|c|c|c|c|c|}
\hline \multirow[t]{2}{*}{ No. } & \multicolumn{2}{|c|}{ Freq. } & \multirow{2}{*}{ Semantic Relations } & \multirow[t]{2}{*}{ Examples } \\
\hline & Of & $\mathbf{S}$ & & \\
\hline$\overline{11}$ & 36 & 220 & POSSESSION & "Mary's book" \\
\hline 2 & 25 & 61 & KINSHIP & "Mary's brother" \\
\hline 3 & 109 & 75 & PROPERTY & "John's coldness" \\
\hline 4 & 11 & 123 & AGENT & "investigation of the crew" \\
\hline 5 & 5 & 109 & TIME-EVENT & "qast year's exhibition" \\
\hline 6 & 30 & 7 & DEPICTION-DEPICTED & "a picture of my nice" \\
\hline 7 & 328 & 114 & PART-WHOLE & "the girl's mouth" \\
\hline 8 & 0 & 0 & HYPERNYMY (IS-A) & "city of Dallas" \\
\hline 9 & 0 & 0 & ENTAILMENT & N/A \\
\hline 10 & 10 & 3 & CAUSE & "death of cancer" \\
\hline 11 & 11 & 62 & MAKE/PRODUCE & "maker of computer" \\
\hline 12 & 0 & 0 & INSTRUMENT & N/A \\
\hline 13 & 32 & 46 & LOCATION/SPACE & "university of Texas" \\
\hline 14 & 0 & 0 & PURPOSE & N/A \\
\hline 15 & 56 & 33 & SOURCE/FROM & "president of Bolivia" \\
\hline 16 & 70 & 5 & TOPIC & "museum of art" \\
\hline 17 & $\overline{0}$ & 0 & MANNER & N/A \\
\hline 18 & 0 & 0 & MEANS & "service of bus" \\
\hline 19 & 10 & 4 & ACCOMPANIMENT & "solution of the problem" \\
\hline 20 & 1 & 2 & EXPERIENCER & "victim of lung disease" \\
\hline 21 & 49 & 41 & RECIPIENT & "Iosephine's reward" \\
\hline 22 & 0 & 0 & FREQUENCY & N/A \\
\hline 23 & 0 & 0 & INFLUENCE & N/A \\
\hline 24 & 5 & 2 & ASSOCIATED WITH & "contractors of shipyard" \\
\hline 25 & 115 & 1 & MEASURE & "qundred of dollars" \\
\hline 26 & 0 & 0 & SYNONYMY & N/A \\
\hline 27 & 0 & 0 & ANTONYMY & N/A \\
\hline 28 & 0 & 0 & PROB. OF EXISTENCE & N/A \\
\hline 29 & 0 & 0 & POSSIBILITY & N/A \\
\hline 30 & 0 & 0 & $\begin{array}{l}\text { CERTAINTY } \\
\end{array}$ & N/A \\
\hline 31 & 120 & 50 & THEME & "acquisition of the holding" \\
\hline 32 & 8 & 2 & RESULT & "result of the review" \\
\hline 33 & 0 & 0 & STIMULUS & N/A \\
\hline 34 & 0 & 0 & EXTENT & N/A \\
\hline 35 & $\overline{0}$ & 0 & $\begin{array}{l}\text { PREDICATE } \\
\end{array}$ & N/A \\
\hline 36 & 107 & 49 & OTHER & "state of emergency" \\
\hline
\end{tabular}

Table 1: The distribution of the semantic relations in the annotated corpus of 20,000 sentences.

on their semantics. The largest subsets did not cover more than $3 \%$ of the OTHER set of examples. This observation shows that the set of 35 semantic relations from Table 1 is representative for genitives.

Table 1 also shows the semantic preferences of each genitive form. For example, POSSESSION, KINSHIP, and some kinds of PART-WHOLE relations are most of the time encoded by the s-genitive, while some specific PART-WHOLE relations, such as "dress of silk" and "array of flowers", cannot be encoded but only by the of-genitive. This simple analysis leads to the important conclusion that the two constructions must be treated separately as their semantic content is different. This observation is also consistent with other recent work in linguistics on the grammatical variation of the English genitives (Stefanowitsch, 2001).

\section{The Model}

\subsection{Problem Formulation}

Given a genitive, the goal is to develop a procedure for the automatic labeling of the semantic relation it conveys. The semantic relation derives from the 
semantics of the noun phrases participating in genitives as well as the surrounding context.

Semantic classification of syntactic patterns in general can be formulated as a learning problem. This is a multi-class classification problem since the output can be one of the semantic relations in the set. We cast this as a supervised learning problem where input/ output pairs are available as training data.

An important first step is to map the characteristics of each genitive construction into a feature vector. Let's define with $\mathbf{x}_{i}$ the feature vector of an instance $i$ and let $X$ be the space of all instances; i.e. $\mathbf{x}_{i} \in X$. The multi-class classification is performed by a function that maps the feature space $X$ into a semantic space $S$

$F: X \rightarrow S$, where $S$ is the set of semantic relations from Table 1, i.e. $r_{k} \in S$.

Let $T$ be the training set of examples or instances $T=\left(\mathbf{x}_{1} r_{1}, \mathbf{x}_{2} r_{2}, \ldots, \mathbf{x}_{n} r_{n}\right) \subseteq(X \times S)^{n}$ where $n$ is the number of examples $\mathbf{x}$ each accompanied by its semantic relation label $r$. The problem is to decide which semantic relation $r$ to assign to a new, unseen example $\mathbf{x}_{n+1}$. In order to classify a given set of examples (members of $X$ ), one needs some kind of measure of the similarity (or the difference) between any two given members of $X$.

\subsection{Feature Space}

An essential aspect of our approach below is the word sense disambiguation (WSD) of the noun. Using a state-of-the-art open-text WSD system with $70 \%$ accuracy for nouns (Novischi et al., 2004), each word is mapped into its corresponding WordNet 2.0 sense. The disambiguation process takes into account surrounding words, and it is through this process that context gets to play a role in labeling the genitives' semantics.

So far, we have identified and experimented with the following NP features:

1. Semantic class of head noun specifies the WordNet sense (synset) of the head noun and implicitly points to all its hypernyms. It is extracted automatically via a word sense disambiguation module. The genitive semantics is influenced heavily by the meaning of the noun constituents. For example: "child's mother" is a KINSHIP relation where as "child's toy" is a POSSESSION relation.

2. Semantic class of modifier noun specifies the
WordNet synset of the modifier noun. The following examples show that the semantic of a genitive is also influenced by the semantic of the modifier noun; "Mary's apartment" is a POSSESSION relation, and "apartment of New York" is a LOCATION relation.

The positive and negative genitive examples of the training corpus are pairs of concepts of the format:

$$
\begin{gathered}
<\text { modifier_semclass\#WNsense; } \\
\text { head_semclass\#WNsense; target>, }
\end{gathered}
$$

where target is a set of at least one of the 36 semantic relations. The modifier_semclass and head_semclass concepts are WordNet semantic classes tagged with their corresponding WordNet senses.

\subsection{Semantic Scattering Learning Model}

For every pair of $<$ modifier - head $>$ noun genitives, let us define with $f_{i}^{m}$ and $f_{j}^{h}$ the WordNet 2.0 senses of the modifier and head respectively. For convenience we replace the tuple $<f_{i}^{m}, f_{j}^{h}>$ with $f_{i j}$. The Semantic Scattering Model is based on the following observations:

Observation 1. $f_{i}^{m}$ and $f_{j}^{h}$ can be regarded as nodes on some paths that link the senses of the most specific noun concepts with the top of the noun hierarchies.

Observation 2. The closer the pair of noun senses $f_{i j}$ is to the bottom of noun hierarchies the fewer the semantic relations associated with it; the more general $f_{i j}$ is the more semantic relations.

The probability of a semantic relation $r$ given feature pair $f_{i j}$

$$
P\left(r \mid f_{i j}\right)=\frac{n\left(r, f_{i j}\right)}{n\left(f_{i j}\right)},
$$

is defined as the ratio between the number of occurrences of a relation $r$ in the presence of feature pair $f_{i j}$ over the number of occurrences of feature pair $f_{i j}$ in the corpus. The most probable relation $\hat{r}$ is

$$
\hat{r}=\operatorname{argmax}_{r \in R} P\left(r \mid f_{i j}\right)
$$

From the training corpus, one can measure the quantities $n\left(r, f_{i j}\right)$ and $n\left(f_{i j}\right)$. Depending on the level of abstraction of $f_{i j}$ two cases are possible:

Case 1. The feature pair $f_{i j}$ is specific enough such that there is only one semantic relation $r$ for which 
$P\left(r \mid f_{i j}\right)=1$ and 0 for all the other semantic relations.

Case 2. The feature pair $f_{i j}$ is general enough such that there are at least two semantic relations for which $P\left(r \mid f_{i j}\right) \neq 0$. In this case equation (2) is used to find the most appropriate $\hat{r}$.

Definition. A boundary $G^{*}$ in the WordNet noun hierarchies is a set of synset pairs such that :

a) for any feature pair on the boundary, denoted $f_{i j}^{G^{*}} \in G^{*}, f_{i j}^{G^{*}}$ maps uniquely into only one relation $r$, and

$b)$ for any $f_{i j}^{u} \succ f_{i j}^{G^{*}}, f_{i j}^{u}$ maps into more than one relation $r$, and

c) for any $f_{i j}^{l} \prec f_{i j}^{G^{*}}, f_{i j}^{l}$ maps uniquely into a semantic relation $r$. Here relations $\succ$ and $\prec$ mean "semantically more general" and "semantically more specific" respectively. This is illustrated in Figure 1.

Observation 3. We have noticed that there are more concept pairs under the boundary $G^{*}$ than above, i.e. $\left|\left\{f_{i j}^{l}\right\}\right| \gg\left|\left\{f_{i j}^{u}\right\}\right|$.

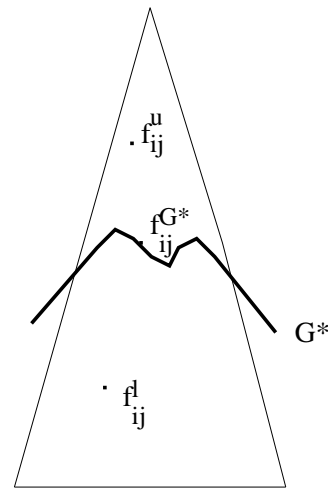

(a)

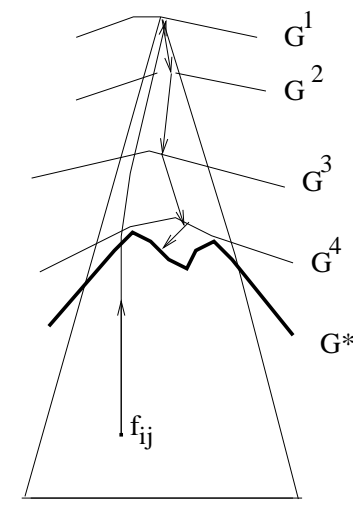

(b)
Figure 1: (a) Conceptual view of the noun hierarchies separated by the boundary $G^{*}$; (b) Boundary $G^{*}$ is found through an iterative process called "semantic scattering".

\subsection{Boundary Detection Algorithm}

An approximation to boundary $G^{*}$ is found using the training set through an iterative process called semantic scattering. We start with the most general boundary corresponding to the nine noun WordNet hierarchies and then specialize it based on the training data until a good approximation is reached.

Step 1. Create an initial boundary
The initial boundary denoted $G^{1}$ is formed from combinations of the nine WordNet hierarchies: abstraction\#6, act\#2, entity\#1, event\#1, group\#1, possession\#2, phenomenon\#1, psychological_feature\#1, state\#4. To each training example a corresponding feature $f_{i j}=<f_{i}^{m}, f_{j}^{h}>$ is first determined, after which is replaced with the most general corresponding feature consisting of top WordNet hierarchy concepts denoted with $f_{i j}^{1}$. For instance, to the example "apartment of the woman" it corresponds the general feature entity\#1entity\#1 and POSSESSION relation, to "husband of the woman" it corresponds entity\#1-entity\#1 and KINSHIP relation, and to "hand of the woman" it corresponds entity\#1-entity\#1 and PART-WHOLE relation. At this high level $G^{1}$, to each feature pair $f_{i j}^{1}$ it corresponds a number of semantic relations. For each feature, one can determine the most probable relation using equation (2). For instance, to feature entity\#1-entity\#1 there correspond 13 relations and the most probable one is the PART-WHOLE relation as indicated by Table 2 .

\section{Step 2. Specialize the boundary}

\subsection{Constructing a lower boundary}

This step consists of specializing the semantic classes of the ambiguous features. A feature $f_{i j}^{k}$ on boundary $G^{k}$ is ambiguous if it corresponds to more then one relation and its most relevant relation has a conditional probability less then 0.9 . To eliminate non-important specializations, we specialize only the ambiguous classes that occurs in more than $1 \%$ of the training examples.

The specialization procedure consists of first identifying features $f_{i j}^{k}$ to which correspond more than one semantic relation, then replace these features with their hyponyms synsets. Thus one feature breaks into several new specialized features. The net effect is that the semantic relations that were attached to $f_{i j}^{k}$ will be "scattered" across the new specialized features. This process continues till each feature will have only one semantic relation attached. Each iteration creates a new boundary, as shown in Figure 1. Table 3 shows statistics of semantic features $f_{i j}^{k}$ for each level of specialization $G^{k}$. Note the average number of relations per feature decreasing asymptotically to 1 as $k$ increases.

\subsection{Testing the new boundary}




\begin{tabular}{|c|c|c|c|c|c|c|c|c|c|c|c|c|c|c|}
\hline $\mathbf{R}$ & $\mathbf{1}$ & $\mathbf{2}$ & $\mathbf{3}$ & $\mathbf{6}$ & $\mathbf{7}$ & $\mathbf{1 1}$ & $\mathbf{1 3}$ & $\mathbf{1 5}$ & $\mathbf{1 6}$ & $\mathbf{1 9}$ & $\mathbf{2 1}$ & $\mathbf{2 4}$ & $\mathbf{2 5}$ & $\mathbf{0 t h e r s}$ \\
\hline
\end{tabular}

Table 2: Sample row from the conditional probability table where the feature pair is entity-entity. The numbers in the top row identify the semantic relations (as in Table 1).

\begin{tabular}{|c|c|c|c||c|c|c|}
\hline \multirow{2}{*}{ Boundary } & \multicolumn{3}{|c|}{ Of-genitives } & \multicolumn{3}{c|}{ S-genitives } \\
\cline { 2 - 7 } & $\mathbf{G}^{\mathbf{1}}$ & $\mathbf{G}^{2}$ & $\mathbf{G}^{3}$ & $\mathbf{G}^{\mathbf{1}}$ & $\mathbf{G}^{2}$ & $\mathbf{G}^{3}$ \\
\hline \hline $\begin{array}{c}\text { Number of modifi er } \\
\text { features }\end{array}$ & 9 & 31 & 74 & 9 & 37 & 31 \\
\hline $\begin{array}{c}\text { Number head } \\
\text { features }\end{array}$ & 9 & 34 & 66 & 9 & 24 & 36 \\
\hline No. of feature pairs & 63 out of 81 & 216 out of 1054 & 314 out of 4884 & 41 of 81 & 157 out of 888 & 247 out of 3276 \\
\hline $\begin{array}{c}\text { Number of features } \\
\text { with only one relation }\end{array}$ & 26 & 153 & 281 & 14 & 99 & 200 \\
\hline $\begin{array}{c}\text { Average number of } \\
\text { relations per feature }\end{array}$ & 3 & 1.46 & 1.14 & 3.59 & 1.78 & 1.36 \\
\hline
\end{tabular}

Table 3: Statistics for the semantic class features by level of specialization.

The new boundary is more specific then the previous boundary and it is closer to the ideal boundary. However, we do not know how well it behaves on unseen examples and we are looking for a boundary that classifies with a high accuracy the unseen examples. We test the boundary on unseen examples. For that we used $10 \%$ of the annotated examples (different from the $10 \%$ of the examples used for testing) and compute the accuracy (f-measure) of the new boundary on them.

If the accuracy is larger than the previous boundary's accuracy, we are converging toward the best approximation of the boundary and thus we should repeat Step 2 for the new boundary.

If the accuracy is lower than the previous boundary's accuracy, the new boundary is too specific and the previous boundary is a better approximation of the ideal boundary.

For the automatic detection of the semantic relations encoded by genitives, the boundary constructed by the Semantic Scattering model is more apppropriate than a "tree cut", like the ones used for verb disambiguation (McCarthy, 1997) (Li and Abe, 1998) and constructed using the Minimum Description Length model (Rissanen, 1978). The developement of a "tree cut" model for the detection of the semantic relations encoded by genitives involves the construction of a different "tree cut" for each head noun and threfore the usage of these cuts is restricted to those head nouns. On the other hand, Semantic Scattering constructs only one boundary that, unlike the "tree cut" model, is general enough to classify any genitive construction, including the ones with constituents unseen during training.

\section{Semantic Relations Classification Algorithm}

The ideal boundary $G^{*}$ is used for classifying the semantic relations encoded by genitives. The algorithm consists of:

Step 1. Process the sentence. Perform Word Sense Disambiguation and syntactic parsing of the sentence containing the genitive.

Step 2. Identify the head and modifier noun concepts.

Step 3. Identify the feature pair. Using the results from WSD and WordNet noun hierarchies, map the head and modifier concepts into the corresponding classes from the boundary and identify a feature pair $f_{i j}$ that has the closest euclidean distance to the two classes.

Step 4. Find the semantic relation. Using the feature $f_{i j}$, determine the semantic relation that corresponds to that feature on the boundary. If there is no such relation, mark it as OTHER.

\section{Results}

For testing, we considered $20 \%$ of the annotated examples. We used half of the examples for detecting the boundary $G^{*}$ and half for testing the system.

\section{$G^{*}$ Boundary Detection}

The algorithm ran iteratively performing boundary 


\begin{tabular}{|c|c|c|c||c|c||c|}
\hline \multirow{2}{*}{ Results } & \multicolumn{3}{|c|}{ Of-genitives } & \multicolumn{3}{c|}{ S-genitives } \\
\cline { 2 - 7 } & Baseline1 & Baseline2 & Results & Baseline1 & Baseline2 & Results \\
\hline \hline $\begin{array}{c}\text { Number of correctly } \\
\text { retrieved relations }\end{array}$ & 49 & 59 & $\mathbf{8 1}$ & 15 & 27 & $\mathbf{7 1}$ \\
\hline $\begin{array}{c}\text { Number of relations } \\
\text { retrieved }\end{array}$ & 73 & 75 & $\mathbf{9 9}$ & 63 & 66 & $\mathbf{8 5}$ \\
\hline $\begin{array}{c}\text { Number of correct } \\
\text { relations }\end{array}$ & 104 & 104 & $\mathbf{1 0 4}$ & 96 & 96 & $\mathbf{9 6}$ \\
\hline \hline Precision & $67.12 \%$ & $76.62 \%$ & $\mathbf{8 1 . 8 2 \%}$ & $23.81 \%$ & $40.91 \%$ & $\mathbf{8 3 . 5 3 \%}$ \\
\hline Recall & $47.12 \%$ & $56.73 \%$ & $\mathbf{7 7 . 8 8 \%}$ & $15.63 \%$ & $28.13 \%$ & $\mathbf{7 3 . 9 6 \%}$ \\
\hline \hline F-measure & $55.37 \%$ & $65.92 \%$ & $\mathbf{7 9 . 8 0 \%}$ & $18.87 \%$ & $33.34 \%$ & $\mathbf{7 8 . 4 5 \%}$ \\
\hline
\end{tabular}

Table 4: Overall results for the semantic interpretation of genitives

specializations on the WordNet IS-A noun hierarchies in order to eliminate the ambiguities of the training examples. Boundary $G^{1}$ corresponds to the semantic classes of the nine WordNet noun hierarchies and boundaries $G^{2}$ and $G^{3}$ to their subsequent immediate hyponyms. For both s-genitives and of-genitives, boundary $G^{2}$ was more accurate then boundary $G^{1}$ and therefore we repeated Step 2. However, boundary $G^{3}$ was less accurate then boundary $G^{2}$ and thus boundary $G^{2}$ is the best approximation of the ideal boundary.

\section{Semantic Relations Classification}

Table 4 shows the results obtained when classifying the 36 relations (the 36 th relation being OTHER) for of-genitives and s-genitives. The results are presented for the Semantic Scattering system that uses $G^{2}$ as the best approximation of the $G^{*}$ together with two baselines. Baselinel system obtained the results without any word sense disambiguation (WSD) feature, i.e. using only the default sense number 1 for the concept pairs, and without any specialization. Baseline 2 system applied two iterations of the boundary detection algorithm but without any word sense disambiguation.

Overall, the Semantic Scattering System achieves an $81.82 \%$ precision and $77.88 \%$ recall for ofgenitives and an $83.53 \%$ precision and $73.96 \%$ recall for s-genitives.

Both the WSD and the specialization are important for our system as indicated by the Baseline systems performance. The impact of specialization on the f-measure (Baseline2 minus Baseline1) is $10.55 \%$ for of-genitives and $14.47 \%$ for s-genitives, while the impact of WSD (final result minus Baseline2) is $14 \%$ for of-genitives and $45.11 \%$ for sgenitives.

\section{Error Analysis}

An important way of improving the performance of a system is to perform a detailed error analysis of the results. We have analyzed the various error sources encountered in our experiments and summarized the results in Table 5.

\begin{tabular}{|c|c|c|}
\hline Error Type & $\begin{array}{c}\text { Of-genitives } \\
\text { \%Error }\end{array}$ & $\begin{array}{c}\text { S-genitives } \\
\text { \%Error }\end{array}$ \\
\hline \hline Missing feature & 28.57 & 29.17 \\
\hline General semantic classes & 28.57 & 20.83 \\
\hline WSD System & 19.05 & 29.17 \\
\hline Reversed order of constituents & 14.29 & 12.5 \\
\hline Named Entity Recognizer & 4.76 & 8.33 \\
\hline Missing WordNet sense & 4.76 & 0 \\
\hline
\end{tabular}

Table 5: The error types encountered on the testing corpus.

\section{Comparison with other Models}

To evaluate our model, we have conducted experiments with other frequently used machine learning models, on the same dataset, using the same features. Table 6 shows a comparison between the results obtained with the Semantic Scattering algorithm and the decision trees (C5.0, http://www.rulequest.com/see5-info.html), the naive Bayes model (jBNC, Bayesian Network Classifier Toolbox, http://jbnc.sourceforge.net), and Support Vector Machine (libSVM, ChihChung Chang and Chih-Jen Lin. 2004. LIBSVM: a Library for Support Vector Machines, http://www.csie.ntu.edu.tw/ cjlin/papers/libsvm.pdf). The reason for the superior performance of Semantic Scattering is because the classification of genitives is feature poor relying only on the semantics of the noun components, and the other 
three models normally work better with a larger set of features.

\begin{tabular}{|c|c|c|}
\hline Accuracy & Of-genitives & S-genitives \\
\hline \hline Semantic Scattering & $79.85 \%$ & $78.75 \%$ \\
\hline Decision Trees (C5.0) & $40.60 \%$ & $47.0 \%$ \\
\hline Naive Bayes (JBNC) & $42.31 \%$ & $43.7 \%$ \\
\hline SVM (LibSVM) & $31.45 \%$ & $23.51 \%$ \\
\hline
\end{tabular}

Table 6: Accuracy performance of four learning models on the same testing corpus.

\section{Discussion and Conclusions}

The classification of genitives is an example of a learning problem where a tailored model outperforms other generally applicable models.

This paper presents a model for the semantic classification of genitives. A set of 35 semantic relations was identified, and we provided statistical evidence that when it comes to genitives, some relations are more frequent than others, while some are absent. The model relies on the semantic classes of noun constituents. The algorithm was trained and tested on 20,000 sentences containing 2,249 of-genitives and 1006 s-genitives and achieved an average precision of $82 \%$, a recall of $76 \%$, and an f-measure of $79 \%$. For comparison, we ran a C5.0 learning system on the same corpus and obtained $40.60 \%$ accuracy for of-genitives and $47 \%$ for s-genitives. A similar experiment with a Naive Bayes learning system led to $42.31 \%$ accuracy for of-genitives and $43.7 \%$ for s-genitives. The performance with a Support Vector Machines learner was the worst, achieving only a $31.45 \%$ accuracy for of-genitives and $23.51 \%$ accuracy for s-genitives. We have also identified the sources of errors which when addressed may bring further improvements.

\section{References}

Barker, Chris. 1995. Possessive Descriptions. CSLI Publications, Standford, CA.

Berland, Matthew and Eugene Charniak. 1999. Finding parts in very large. In Proceeding of ACL 1999.

Fellbaum, Christiane. 1998. WordNet - An Electronic Lexical Databases. Cambridge MA: MIT Press.
Girju, Roxana, Adriana Badulescu, and Dan Moldovan. 2003. Learning semantic constraints for the automatic discovery of part-whole relations. In Proceedings of the HLT-NAACL 2003.

Gildea, Daniel and Daniel Jurafsky. 2002. Automatic Labeling of Semantic Roles. Computational Linguistics, 28(3):277-295.

Hearst, Marti. 1998. Automated Discovery of WordNet relations. In An Electronic Lexical Database and Some of its Applications. MIT Press, Cambridge MA.

Lapata, Maria. 2000. Automatic Interpretation of Nominalizations. In Proceedings of AAAI 2000, 716-721.

Li, Hang and Naoki Abe. 1998. Generalizing case frames using a thesaurus and the mdl principle. Computational Linguistics, 24(2):217-224.

McCarthy, Diana. 1997. Word sense disambiguation for acquisition of selectional preferences. In Proceedings of the ACL/EACL 97.

Moldovan, Dan, Adriana Badulescu, Marta Tatu, Daniel Antohe, and Roxana Girju. 2004. Models for the Semantic Classifi cation of Noun Phrases. In Proceedings of the HLT-NAACL 2004, Computational Lexical Semantics Workshop.

Nikiforidou, Kiki. 1991. The meanings of the genitive: A case study in the semantic structure and semantic change. Cognitive Linguistics, 2:149-205.

Novischi, Adrian, Dan Moldovan, Paul Parker, Adriana Badulescu, and Bob Hauser. 2004. LCC's WSD systems for Senseval 3. In Proceedings of Senseval 3.

Rissanen, Jorma. 1978. Modeling by shortest data description. Automatic, 14.

Rosario, Barbara and Marti Hearst. 2001. Classifying the Semantic Relations in Noun Compounds via a Domain-Specifi c Lexical Hierarchy. In Proceeding of EMNLP 2001.

Siegel, S. and N.J. Castellan. 1988. Non Parametric Statistics for the behavioral sciences. New York: McGraw-Hill.

Stefanowitsch, Anatol. 2001. Constructional semantics as a limit to grammatical alternation: Two genitives of English. Determinants of Grammatical Variation in English.

Taylor, John. 1996. Possessives in English. An exploration in cognitive grammar. Oxford, Clarendon Press.

Vikner, Carl and Per Anker Jensen. 1999. A semantic analysis of the English genitive: interaction of lexical and formal semantics. 